\title{
THE SEIBT DIRECT INDICATING WAVE METER.*
}

\author{
By Emil J. Simon and Dr. Alfred N. Goldsmith
}

A conducting ring placed in front of a coil in which flows an alternating current will be repelled from the coil. The cause of the repulsion is the interaction of the current induced in the ring by the alternating magnetic field in which it is placed, and the magnetic field itself. This repulsion is known as the Thomson (1) or dynamometer effect. The exact magnitude of the effect, and the conditions under which it is most readily produced and measured, can be ascertained from the articles mentioned in bibliography at the end of this article. The industrial applications of the dynamometer effect have been many. In a braking device on induction motors and in alternating current wattmeters it has been widely utilized. It has even been employed by Mandelstam and Papalexi (2) in a special form of double, alternating current, mirror galvanometer whereby the radio frequencies can be very precisely measured. Tho measurements of wave length by the method of Mandelstam and Papalexi are of a high order of precision, the convenience of their method is hardly as great as that of the familiar method of Bjerknes, and it is not well suited for use except under laboratory conditions. A pointer and scale instrument of this type would be difficult to construct. It has remained for Dr. George Seibt to place on the market a wave meter based on the Thomson effect, portable, and direct indicating. We shall describe this instrument in further detail.

As shown in Figure 1, when an alternating current passes thru the coil $\mathrm{S}$, the ring $\mathrm{R}$ will be repelled. If two coils, $\mathrm{S}_{1}$ and $\mathrm{S}_{2}$ (Figure 2), be placed on opposite sides of the ring $\mathrm{R}$, they will exert on this ring the opposing forces $\mathrm{K}_{1}$ and $\mathrm{K}_{2}$. If the ring is free to move it will assume an intermediate position of equilibritum between the two coils. If the two coils are alike,

(1) Elihu Thomson, Electrical World, May 28, 1887.

(2) Mandelstam and Papalexi, Annalen der Physik, 1910, Vol. 33, Page 490.

*Partially based on Dr. George Seibt's French Patent No. 446,251 and English Patent No. 16,874. 


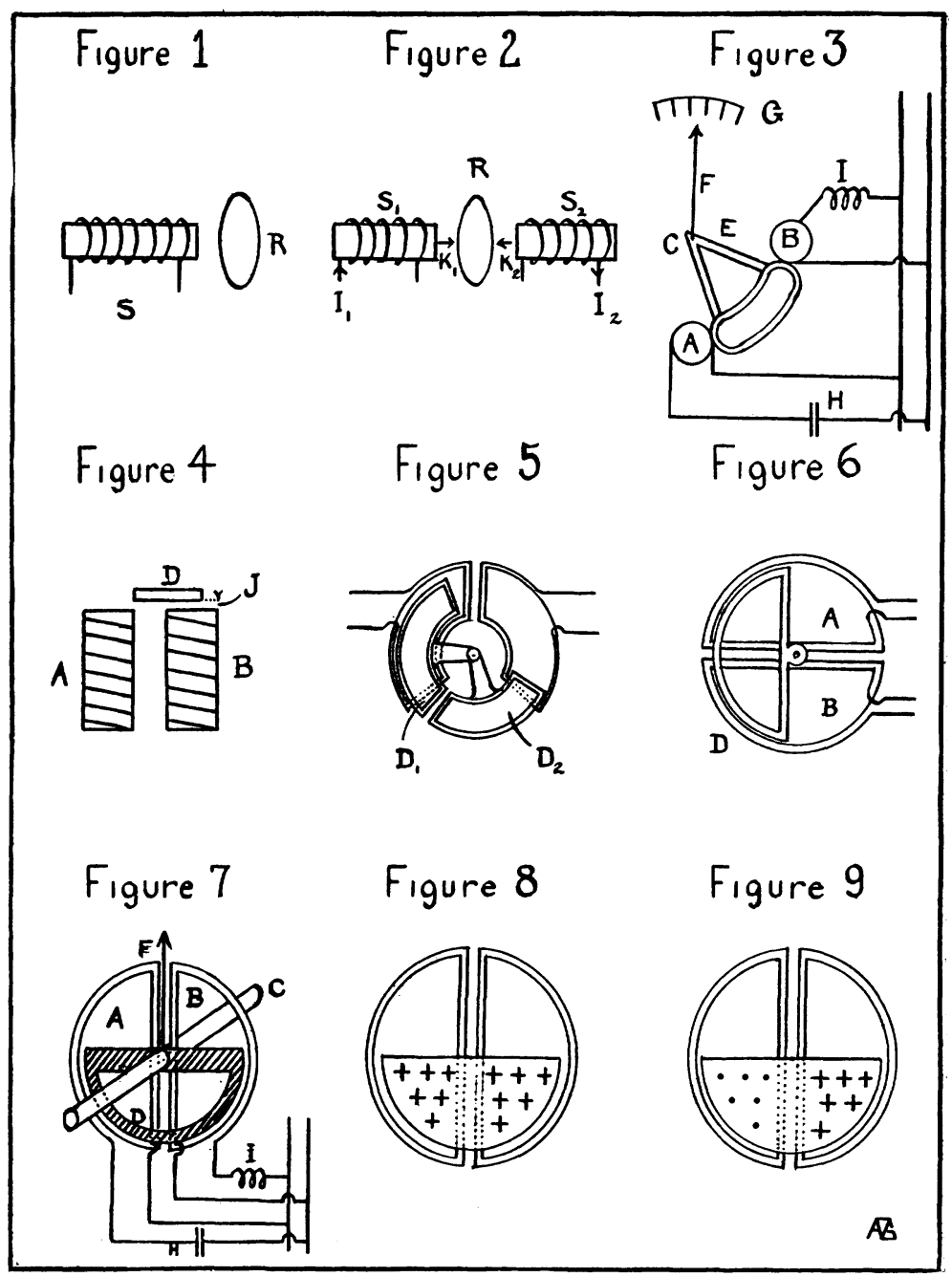


the currents thru them equal, and if no external control force acts on the ring, it will place itself exactly half way between the coils. But if the currents are unequal, the ring will move toward the coil carrying the smaller current. The problem of constructing a wave meter on this basis then resolves itself into the following: What arrangement of circuits connected to the coils $S_{1}$ and $S_{2}$ will ensure a division of the total current between them, such that the relation between the currents in $S_{1}$ and $S_{2}$ shall be dependent only on the frequency of the total current, but not on its magnitude?

A number of methods of constructing the instrument will be given. Referring to Figures 3 and 4, coils $\mathrm{A}$ and $\mathrm{B}$ are placed approximately $90^{\circ}$ apart relative to the axis $\mathrm{C}$ of the instrument. Over them is mounted on the frame $\mathrm{E}$ the armature $\mathrm{D}$, consisting of a piece of metal forming a closed circuit or of a number of short-circuited windings. Both armature and frame are free to rotate about the axis $\mathrm{C}$, carrying with them the pointer $\mathrm{F}$, which moves over the graduated scale G. It will be seen that this arrangement is equivalent to that shown in Figure 2, inasmuch as an alternating current thru either coil A or B will tend to repel the armature $\mathrm{D}$ toward the other coil. As before, the stable position of the amature is determined by the relation between the currents in the coils A and B or the fields due to these currents.

The variation of the fields or of the currents with the frequency may be obtained in various ways other than that mentioned above, which was finally adopted. Thus the coil A may be connected to the circuit the frequency of which is to be determined thru the condenser $\mathrm{H}$, and the coil $\mathrm{B}$ thru the inductance $\mathrm{I}$.

The form of armature construction shown permits of placing it very close to the coils A and B. The closeness of coupling between the coils and the armature can be varied by altering the distance $\mathrm{J}$.

The sensitiveness of the instrument and also the operative angular range on the graduated scale are increased if the surface enclosed by the armature, and also the surface enclosed by each of the coils $\mathrm{A}$ and $\mathrm{B}$, subtends a greater angle at the center than $90^{\circ}$. In Figure 5 is shown an arrangement in which the surface of the armature and coils of the instrument are angularly measured, and relative to the axis of the instrument, 
each greater than $90^{\circ}$. The armature is here split into two parts $\mathrm{D}_{1}$ and $\mathrm{D}_{2}$. The theoretical maximum angle of deviation is $120^{\circ}$; but in practice, owing to the weakening of the forces of deflection at larger angles of deflection and the consequent inaccuracy in the readings of the instrument, deflections greater than about $60^{\circ}$ are not used. The arrangement shown in Figures 6 and 7 , in which the surface of the armature and fixed coils are, angularly measured relative to the axis of the instrument, about $180^{\circ}$ wide is still more sensitive. The maximum theoretical angle of deflection reaches $180^{\circ}$, and the maximum angle used in practice about $110^{\circ}$.

Another possible way of constructing the armature is to shape it as part of a cylindrical surface, the axis of which is the axis of the instrument at the pivot. The surface of the armature is then parallel to the axis of the instrument, and the end surfaces of the fixed coils are preferably arranged also to be parallel to this axis.

An interesting phenomenon which has been observed in connection with the arrangement shown in Figure 7 may be here mentioned. If the coils $\mathrm{A}$ and $\mathrm{B}$ are so connected that their fields have one and the same direction and are in phase with each other, the armature does not come to rest in some intermediate position of equilibrium as before. It swings toward the coil carrying the smaller current, and comes to lie entirely in the field of that coil with the pointer thrown against one end of the graduated scale. This condition is shown in Figure 8. The lines of force are represented as circles or crosses, depending on whether they are to be regarded as emerging from the plane of the paper or entering it. If the fields of the fixed coils are equal to each other, the resultant force tending to produce rotation of the armature is zero independently of the position of the latter. There will therefore be no definite position of rest, the movable armature being in what may be called a "floating" state. The above phenomenon is rendered quite explicable when one considers that, in Figure 8 , coils $\mathrm{A}$ and $\mathrm{B}$ are in effect but a single coil; and that the motion of a conductor in an alternating magnetic field is toward regions of least magnetic force. A careful consideration of Figure 9, wherein the fields of the coils A and B are represented as in opposite phase, leads to the conclusion that we may have an equilibrium position in this case. 
The occurrence of the phenomenon of "floating," which may take place even tho large currents are flowing in the armature, would render the instrument practically useless if no means were provided for avoiding it. For example, if we used such an instrument for measuring rapidly alternating currents, the indications would be quite unreliable because the "floating" is caused not by the relation between the absolute values of the currents, but by the relation between the phases of the fields.

Referring again to Figure 7, if the condenser $\mathrm{H}$ is made so small that its equivalent alternating current resistance is greater by a certain amount than that of the coil $\mathrm{A}$, then the currents in the coils $\mathrm{A}$ and $\mathrm{B}$ will have a phase displacement of $180^{\circ}$. Whether the fields will have the same phase displacement as the currents depends on the sense in which the coils are wound, and on the way in which they are connected to the circuit the frequency of which is to be measured. The phase displacement between the fields can always be brought to $180^{\circ}$ by simply interchanging the connections of one of the coils.

Experiments have shown that when the arrangement shown in Figure 7 is used to measure the frequency of damped aiternating currents, as produced for example by spark discharges, the values obtained are very inaccurate (as compared with the true values of the wave lengths.) The cause of this inaccuracy is that an oscillating circuit is formed by the condenser $\mathrm{H}$, the inductances $\mathrm{A}$ and $\mathrm{B}$, and the inductance outside the instrument which serves for coupling purposes. In this circuit free oscillations are induced, these oscillations having in general a different frequency from that to be determined. Calibration of the instrument with sustained alternating current would therefore introduce a continuous error when measuring damped oscillations, the magnitude of this error increasing with the damping of the oscillations, and with the closeness of coupling of the instrument to the circuit of which the frequency is to be measured; since the frequency of the current in the instrument would be a function of the coupling to the exciting circuit.

In order to overcome the serious objection just mentioned, the free oscillations of the instrument are strongly damped. The instrument may even be made completely aperiodic. The damping of the instrument is accomplished by inserting an ohmic resistance in parallel or in series with the condenser, or by entirely dispensing with the condenser. For instance, if the condenser in 
Figure 7 is replaced by a non-inductive resistance, the "free period" error is eliminated.

The insertion of this resistance is unfortunately accompanied by an energy loss. This disadvantage may become so great as to prevent the use of the instrument in radio telegraphy, where small energy losses are essential. For example, a wave meter which consumes 50 watts may be said to be unsuitable for such work since that amount of power would be quite sufficient for signalling over a distance of about 100 kilometers, and, as a loss, could be justified only in very exceptional cases.

The energy loss may be reduced by making the resistance to be inserted in the circuit of the coil A very small. The disadvantage which then arises is that the ratio of the currents flowing thru the fixed coils varies but slightly with the frequency, and the divisions on the scale corresponding to the various frequencies are excessively close. This crowded scale is overcome by the arrangement shown in Figure 10 . Here a resistance $K$ is arranged in series with the fixed coil $A$ and a resistance $L$ in parallel with the fixed coil B. Theory and experiment have shown that by these means a graduated scale with widely spaced divisjons can be obtained, in spite of the fact that the energy consumption is substantially decreased. Another advantage of the arrangement of Figure 10 is that with suitable electrical constants the divisions of the graduated scale can be made substantially equal, so that the deflections are proportional to the wave lengths.

Considering the phase displacements of the fields in the case shown in Figure 10, it is impossible to obtain a displacement of $0^{\circ}$ or $180^{\circ}$, the displacement lying between $0^{\circ}$ and $90^{\circ}$ or between $90^{\circ}$ and $180^{\circ}$, without ever reaching the extreme limits. The "floating" effect previously referred to is observed in the arrangement of Figure 10 but to a smaller extent. It is advisable in consequence to lead the currents thru the fixed coils $A$ and $B$ in such a manner that the phase angle is as great as possible, that is to say between $90^{\circ}$ and $180^{\circ}$. If, after this has been done the connections to one of the coils is reversed, it can be immediately noticed that the directive force is considerably reduced, and that the armature then tends to "float."

The "floating" phenomenon can occur only when the armature has a well defined circuit reaching into the fields of the fixed coils. A definite circuit is missing in the case shown in Figure 11. The armature being a semi-circular solid disc, each of the 


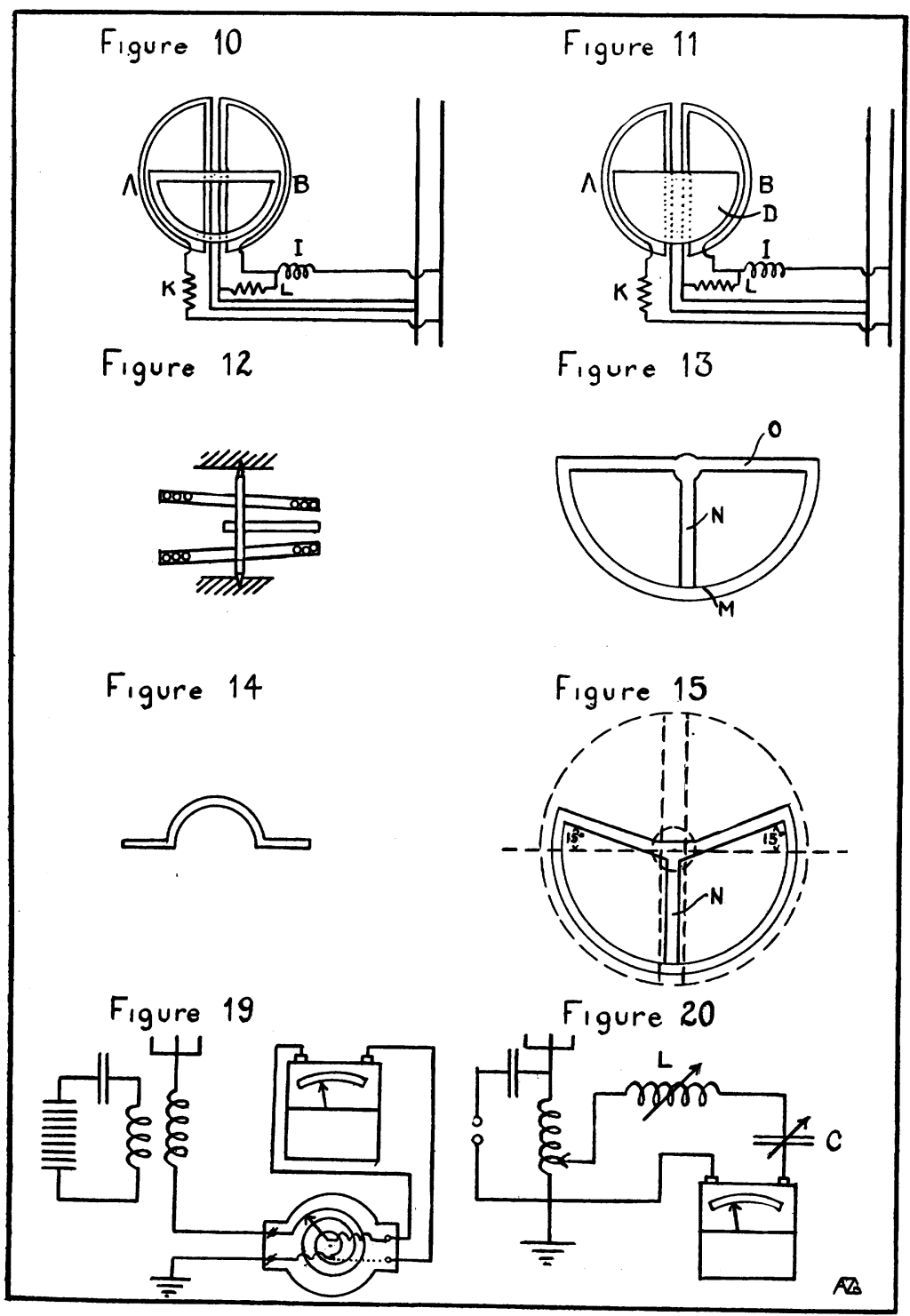


fixed coils will induce in it mainly separate currents. It will be readily seen, however, that this construction is disadvantageous for other reasons. The short-circuited eddy currents, induced by each of the coils, flow within each field radially toward the axis of the instrument and then back toward the periphery with nearly the same strength. The forces produced will nearly neutralize each other, and only a small difference will be left to rotate the armature. It may be stated that the use of a well defined circuit, which is as free as possible from eddy currents, and is under the influence of two fields alternating with a proper phase difference, has been found to be an essential requirement for the success of the instrument. It is further to be noted that if the instrument is constructed in this manner, at most a small current will flow thru the armature after it has reached its position of equilibrium. In the arrangement of Figure 7, no current whatever will flow thru the armature since the phase displacement may be made $180^{\circ}$; and in the arrangement of Figure 10, where this displacement cannot be attained, the armature current can still be made very small by the proper connection to the outside circuit of coils $\mathrm{A}$ and $\mathrm{B}$.

In order to attain the requisite sensitiveness, the movable parts of this instrument must be made very light, and for this reason it is desirable to reduce the current in the armature as far as possible to avoid overheating.

To secure still greater sensitiveness and a still wider scale, the modified construction illustrated in Figure 12 is adopted. It will be seen that the fixed coils 'A and B are mounted slightly inclined to the surface of the armature $\mathrm{D}$. The increased sensitiveness and wider range of the scale are due to the gradient of field intensity, and consequent directive force, found when using this disposition of parts. The condition of equilibrium is that the electromotive forces induced in the armature by the two coils shall be equal and opposite, consequently the angle of rotation will be greater when the field intensity of the coil toward which the armature moves is larger at the entering edge than when it is constant. The inclined coils therefore produce a greater variation in the deflection angle for a definite change in frequency than parallel coils.

To reduce the weight of the armature as far as possible, it has been constructed of sheets of aluminum 0.05 to 0.3 millimeters thick, and this range of thickness has been found satisfactory. 
The disadvantage of the construction shown is that the part $M$ (Figure 13) of the armature is easily bent. To avoid this weakness the sheet is formed with a rib, as shown on an enlarged scale in Figure 14, and an insulated reinforced arm $\mathrm{N}$ is connected between the parts $\mathrm{O}$ and $\mathrm{M}$ of the armature, as shown in Figure 13. Instead of using the rib construction, the sheet may be T-shaped, bent into tubular form, or otherwise suitably reinforced. The reinforcement of the armature is especially required in the case where the armature has a surface angularly greater than that of the fixed coils. (Such an increased angular surface increases the sensitiveness of the instrument. For instance if the surface of the armature is increased $15^{\circ}$ on each side (as in Figure 15), the resistance in series with coil A can be reduced to half its former value without affecting the range of the scale.)

So far as the form of the fixed coils is concerned, it is advantageous to make them as flat as possible in order to bring the windings into close proximity with the armature. It is also preferable to divide each coil into two separate coils arranged one above the other with the armature lying between them, the separate coils being connected in parallel with each other, and not in series. Their arrangement in parallel prevents the production of high potential differences which might give rise to sparks jumping to the armature or from coil to coil. Moreover, this arrangement reduces the total reactance of the instrument and makes it possible to operate it with small potential differences. The connection of the instrument to existing installations is thereby considerably facilitated.

The wave meter is to be connected in the grounded side of the antenna or other circuit in which high potential differences exist. Experiment has shown that electrostatic forces will slightly influence the movable portion of the instrument; so that, in the absence of a ground connection the instrument should be connected to that point of the circuit where a potential node is found. The error which is produced in the wave meter readings by electrostatic forces may be avoided by exciting the instrument not directly, but inductively by means of a transformer.

A further error in the readings may be caused by the action of powerful exterior fields which pass thru the coils of the meter and alter the distribution of the lines of force. However in the case of radio frequency currents this error can be avoided by surounding the working parts of the instrument by a copper or 
aluminum casing; and in the case of low frequency currents preferably by the use of an iron casing.

The scale of the instrument may have more than one range of wave lengths, and the only change necessary in the electrical connections of the instrument in passing from one range to the others is a variation of the non-inductive resistance $K$ of the instrument. This can be readily accomplished by a switch which short circuits a part of the resistance.

In the arrangements so far shown, the fixed coils A and B serve for two functions. Firstly, they induce in the movable armature $\mathrm{D}$ alternating currents. And secondly, they produce the turning force on the armature thru the interaction of their fields and the induced currents in the armature.

One can construct a modification of the instrument, wherein the two functions mentioned above are separately performed by individual coils. The coils $\mathrm{A}$ and $\mathrm{B}$ are semi-circular as before, and serve to induce currents in the armature. They do not, however, exert any appreciable mechanical force on the armature because certain portions $R_{1}$ and $R_{2}$ of the armature are considerably removed from the neighborhood of the coils. In addition extra coils $\mathrm{X}$ and $\mathrm{Y}$ are provided in close proximity to the portions $R_{1}$ and $R_{2}$ of the armature. These coils are intended to produce highly uniform fields, which fields will cause a torque on $R_{1}$ and $R_{2}$. Coils $X$ and $Y$ may be made semi-circular, but are preferably complete circles, for they will then induce no currents in the armature. Adopting such an arrangement, the energy consumed by the instrument is again substantially reduced and the sensitiveness increased, because the number of turns of the coils $\mathrm{A}$ and $\mathrm{B}$ may be considerably increased without in any way increasing the energy consumption. The coils of this instrument, when used for radio frequencies are preferably wound with "litzendraht" or multiply stranded wire.

A number of other applications of the constructions given may be mentioned. The instrument may be used as a relay or controlling device. In this case the pointer connected to the movable armature is arranged to press against fixed contacts whenever definite frequencies are reached. The points of the pivot being mounted in insulating bearings, special means of making contact are necessary. This may be accomplished by connecting a very fine and flexible wire to the pivot or by means of double contacts. The instrument may also be employed as a tachometer 
or indicating speed meter. It is merely necessary to connect its terminals to a small alternator which is belted or directconnected to the rotating machinery, the speed of which is desired. The advantage of this instrument as compared to the usually employed voltmeter in tachometers is that the voltage of the alternator of the tachometer may drop considerably thru weakening of the permanent field magnets (which are usually used) thereby affecting the reading of the usual instrument. And there is no necessity of calibrating the instrument with the particular alternator to which it is to be connected as is the case when a voltmeter is used as the indicator.

It need hardly be said that in the form of meter used for measuring wave lengths in radio telegraphy no iron cores are employed in the coils. Great care is exercised in this instrument to minimise bearing friction and to balance the armature very carefully.

The portable model of the instrument is shown in Figure 16. The terminals of the instrument are seen on the top. To the right is the switch which, by short circuiting a portion of the resistance permits the use of either the upper or lower scale. The upper scale runs from 150 to 1,500 meters, each division being 10 meters and the lower scale from 500 to 3,000 meters, each division corresponding to 100 meters. Directly above the scales is seen an indicating lamp which serves the following purpose. Theoretically the indications of the wave meter are independent of the current flowing, but in practise it is necessary to keep the current between certain major and minor limits. The objections to a very small current thru the instrument are that there will be no certainty that the friction in the pivots exerts no effect on the reading, that slight unbalancing of the armature is not rendered negligible in its effect, and that the slow motion of the pointer to its final position under the action of small currents makes the reading uncertain. To readily ascertain that the current flowing thru the meter has a correct value, the small indicating tungsten lamp above the scale is watched. This lamp is chosen so that the slightest dark red glow indicates the minimum allowable current, and a bright white light the maximum permissible current. It is not important that any particular degree of brightness be obtained, but in order to obtain long life for the lamp it is desirable to work nearer the lower current limit.

As will be seen from the theory of the instrument, its indications are reliable only when there are alternating currents of a single frequency in the exciting circuit. If there are currents of 


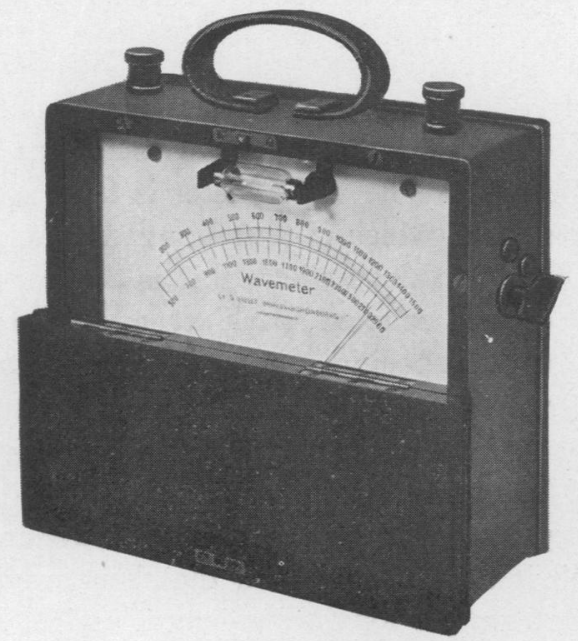

Figure 16

Seibt Direct Reading Wavemeter-1912 Portable Model Range $\left\{\begin{array}{l}\text { Scale I }-300-1500 \mathrm{M} \\ \text { " II-500-3000 M }\end{array}\right.$

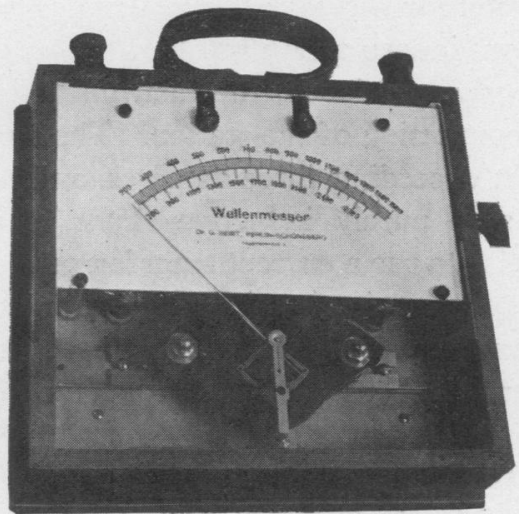

Figure 17

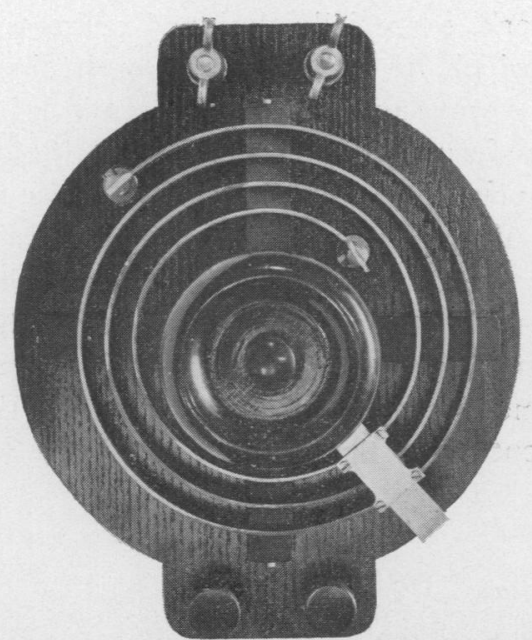

Figure 18 
two or more frequencies present, unless the amplitude of all but one of them are negligibly small, the arrangement shown in Figure 19 must be used. The wave meter is here connected in the resonance circuit $\mathrm{L} \mathrm{C}$ which is tuned until the lamp brilliancy is a maximum. Two positions where this is the case will be found in an ordinary closely coupled spark set, and the readings of the meter at each of these positions gives the wave length of each of the "coupling" waves. Such an arrangement is naturally unnecessary in the case of quenched spark sets where but a single frequency should be present.

The energy consumed by the form of wave meter shown in the photograph is about 4 watts on the short wave length scale, and about 1 watt on the long wave length scale. A switchboard type of the instrument having the same electrical constants is also constructed. For work covering wide ranges of wave length, the instrument may be built with a scale covering from 800 to 4,500 meters in steps of 100 meters. The even spacing of the scale divisions is seen on Figure 16.

As previously mentioned, the instrument is to be connected in the ground connection of the station. The meter is constructed for currents not exceeding three amperes, and it is usually necessary to connect a purely inductive shunt in parallel with the instrument or else to use a current transformer. Since the amount of inductance required in the shunt is dependent on the current flowing in the antenna circuit, it is always best for a first trial reading to use a very small value of the inductance as a shunt. If no glow occurs in the indicating lamp, the current may be increased by increasing the inductance of the shunt and the process continued until a moderate glowing of the lamp is obtained.

Realizing that this process might be somewhat troublesome in practical work, a specially designed current transformer has been devised which, if properly employed, obviates all risk of burning out the wave meter but still permits of rapid manipulation. The transformer is shown in Figure 18, and the means of connecting it in circuit in Figure 19. The entire ring is connected in series with the antenna at the grounded end and the wave meter is connected to a separate pair of terminals, the inductance across which is adjustable. Because of variations in the antenna current such an adjustment is desirable. These inductive couplers for the wave meter are made in two sizes, and the type used depends on the output of the set with which the wave meter is to 
be used. The smaller coupler is for antenna currents of 3 to 30 amperes and is suitable for 2 and 5 kilowatt sets. The larger coupler is intended for use where the antenna current lies between 20 and 100 amperes, and is therefore appropriate for 10 to 50 kilowatt sets.

The advantages of this type of wave meter may be shortly recapitulated: Direct indication of the wave length by a needle moving over a calibrated scale, freedom from adjustment to resonance (unless two waves are present), rapidity of manipulation and reading, readings independent of irregularities in action of spark gap or arc, readings independent of current thru instrument between wide limits, self-contained, light, and rugged construction, long scale, with even divisions, long wave length range with high accuracy by use of two scales on the same instrument.

The instrument weighs 5 lbs. (or 2.3 kilograms).

Its dimensions are $9 "$ by $8^{\prime \prime}$ by $35 / \mathrm{s}^{\prime \prime}$ (or $23 \mathrm{~cm}$. by $21 \mathrm{~cm}$. by $9 \mathrm{~cm}$.).

SUMMARY: A direct indicating wave meter depending for its action on the balancing of the repulsive forces exerted by two fixed coils on a movable ring armature is described. Circuit arrangements are shown whereby the division of current between the coils is made dependent only on the frequency. Electrical and mechanical means for increasing the sensitiveness of the instrument, diminishing its energy absorption, obtaining an even wave length scale, and eliminating the so-called "floating effect" are described in detail. Other industrial applications of the instrument are given.

\section{BIBLIOGRAPHY.*}

J. A. Fleming, The Alternate Current Transformer, Vol. 1, Page 309.

Steinmetz, Alternating Current Phenomena, New York, Page 194.

Rüdenberg, Energie der Wirbelströme, Stuttgart, 1906.

Morck, Theorie der Wechselstromzähler, Stuttgart, 1906.

*On eddy currents, alternating current repulsion, alternating current repulsion measuring instruments. 
E. Wirz, Beitrag zur Theorie und Lntersuchung der Ferrarismessgeräte, Berlin, 1912.

Elihu Thomson, Electrical World, May 28, 1887.

J. A. Fleming, Proc. Royal Inst., Volume 13, Page 311, 1890.

M. Borgman, Comptes Rendus, Feb. 3, 1890, Page 233.

M. Borgman, Comptes Rendus, April 21, 1890, Page 849.

G. T. Walker, Phil. Trans. Royal Soc., Volume 183 A, Page 279, 1892. 599.

J. J. Thomson, London Electrician, 1892, XXVIII, Page

Brugger, Elektrotechnische Zeitschrift, 1895, Page 677

Schrottke, Elektrotechn. Zeitschr., 1901, Page 657.

R. Gans, Zeitschr. für Mathematik und Physik, 1902, Vol. 48, Page 1.

G. W. Pierce, Physical Review, Volume 20, Page 226, April, 1905.

Görner, Schweiz. Elektrotechn. Zeitschr., 1907, Page 617.

David and Simons, Elektrotechn. Zeitschr., 1907, Page 942

Sumpner, Proc. Royal Soc., Series A, Volume 80, Page 310. 1908.

Brückmann, Elektrotechn. Zeitschr., 1910, Page 859.

Görner, Helios, 1910, Vol. 20.

Iliovici, La Lumiére Electrique, 1911, Vol. 19.

Rogowski, Zeitschr. für Elektrotechnik und Maschinenbau, 1911, Vol. 45.

\section{DISCUSSION.}

LEE DE FOREST: Are the indications of this wave meter independent of the applied electromotive force? The currents thru each of the branches must depend on the electromotive force.

EMIL J. SIMON: Dr. Seibt states that theory and practise agree in the conclusion that within certain limits (indicated very well by the glowing of the lamp in the meter) the readings are independent of the applied electromotive force. However, I have found that the readings may be as much as $5 \%$ off if the 